\title{
The Zoo of emission lines in the spectrum of Cir X-1 observed by XMM-Newton
}

\author{
R. Iaria, A. D’Ai', T. Di Salvo, G. Lavagetto and N. R. Robba \\ DSFA-Universita' degli Studi di Palermo
}

\begin{abstract}
We present the preliminary analysis of a $10 \mathrm{ks}$ XMM-Newton EPIC/pn observation of Cir X-1 immediately after the zero phase. The continuum emission is modeled using a blackbody component partially absorbed by neutral matter probably located around the binary system. We detect a forest of emission lines associated to highly ionized ions.
\end{abstract}

Keywords: Photoionization of atoms and ions, Atomic processes and interactions, Atomic and molecular data, spectra, and spectral parameters.

PACS: $32.80 . \mathrm{Fb}, 95.30 . \mathrm{Dr}, 95.30 . \mathrm{Ky}$

\section{INTRODUCTION}

Cir X-1 is a peculiar low-mass X-ray binary (LMXB). The detection of type I X-ray bursts (Tennant et al. 1986a, 1986b) indicates that the compact object is a neutron star. The canonical scenario accepted for Cir X-1 argues that the source has a companion star of 3-5 $\mathrm{M}_{\odot}$ (probably a subgiant; see Johnston et al. 1999), an orbital period of 16.6 days, deduced from the radio and X-ray light curve of the source, and an eccentric orbit with e $\simeq 0.7-0.9$ (Murdin et al. 1980; Tauris et al. 1999).

Using BeppoSAX data, Iaria et al. (2001a) analyzed the 0.1-100 keV spectrum of Cir $\mathrm{X}-1$ at phases $0.11-0.16$. They detected a strong absorption edge at $8.4-8.7 \mathrm{keV}$, produced by highly ionized iron. Iaria et al. (2001b), using ASCA data, studied the energy spectrum of $\mathrm{Cir} \mathrm{X}-1$, along its period, distinguishing three different $\mathrm{X}$-ray states of the source as a function of its phase. At the phases near phase zero, the hydrogen column derived from the edge was $10^{24} \mathrm{~cm}^{-2}$; at larger values of the phase the hydrogen column decreased; the absorption edge (from ionized iron) was no longer detected at phases from 0.78 to 1 , while at these phases a partial covering component was required with an equivalent hydrogen column of $10^{24} \mathrm{~cm}^{-2}$.

Recently two relevant detections interested Cir X-1: 1) the detection of P-Cygni profiles at phase 0.99 (Brandt \& Shulz, 2000; Shulz \& Brandt, 2002). The presence of these features in the spectrum of Cir X-1 indicates that an outflow of matter with a velocity of $2000 \mathrm{~km} / \mathrm{s}$ is present. Assuming that the source is seen nearly edge-on the authors describe a scenario in which the outflow of matter is produced in the outer region of the accretion disc following the prescription of thermally driven wind reported by Begelman et al. (1983); 2) the radio detection of an ultrarelativistic outflows similar to those produced by active galactic nuclei (Fender et al. 2004) with a superluminar motion of the jet with an apparent velocity of $15 c$. The detection of the superluminal motion in the jet of Cir X-1 implies that $\theta$, the angle between the line of sight and the direction of the jet, should be less than $5^{\circ}$. Since the jet should have a direction almost 
perpendicular to the accretion disk it implies that $\mathrm{Cir} \mathrm{X}-1$ is seen face-on.

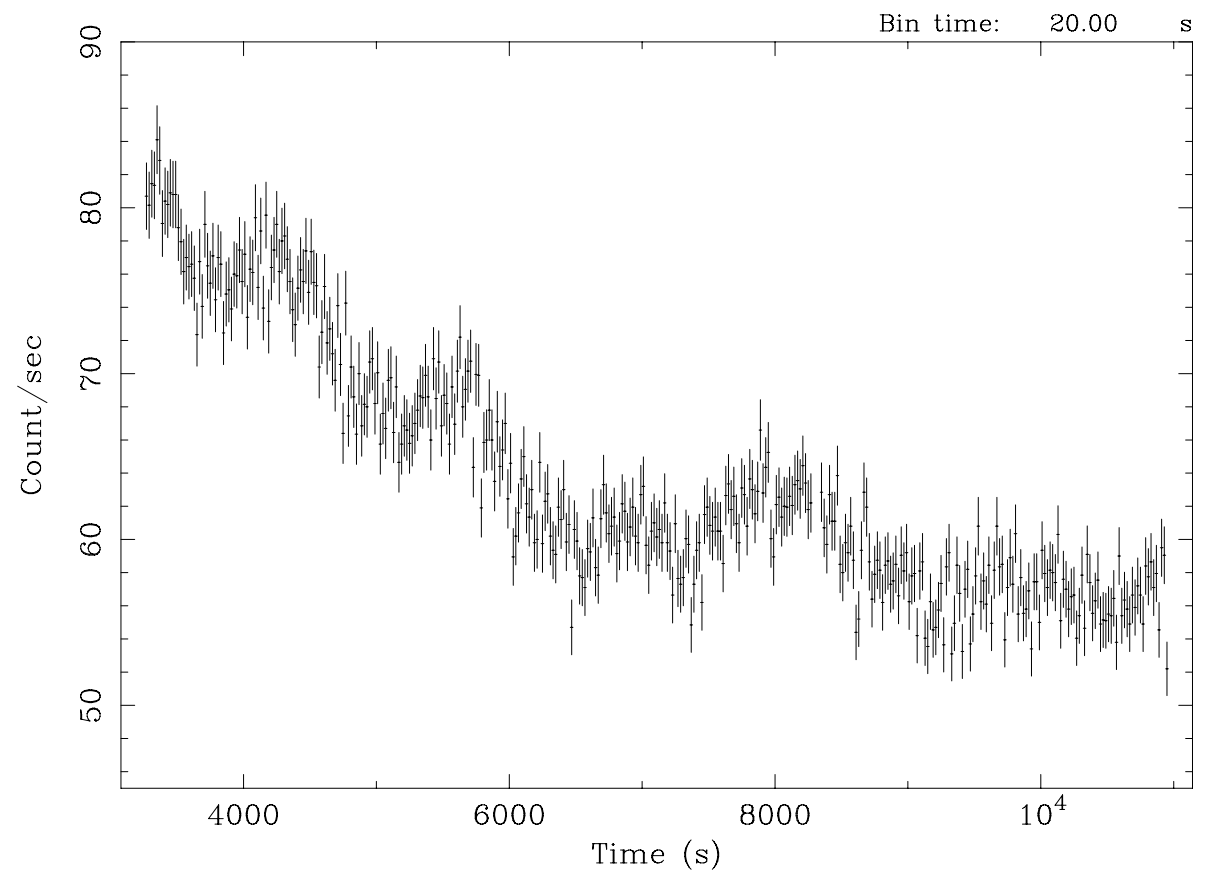

FIGURE 1. EPIC/pn lightcurve of $\mathrm{Cir} \mathrm{X}-1$, the bin time is $20 \mathrm{~s}$.

\section{OBSERVATION AND SPECTRAL ANALYSIS}

The XMM-Newton Observatory (Jansen et al. 2001) includes three $1500 \mathrm{~cm}^{2}$ X-ray telescopes each with an European Photon Imaging Camera (EPIC) at the focus. Two of the EPIC imaging spectrometers use MOS CCDs (Turner et al. 2001) and one uses pn CCDs (Struder et al. 2001). Reflection Grating Spectrometers (RGS, Den Herder et al. 2001) are located behind two of the telescopes. We analyze the $10 \mathrm{ks}$ observation of Cir X-1 taken from the XMM public archive (Obsid 94520201). During the observation only EPIC-pn, operating in Timing mode, and RGS were active. Since we have interest to study the Fe-K region we analyze only the pn data reducing them using the Science Analysis Software (SAS) version 7.0.0 and calibration files released on 16 May 2006. In pn Timing mode, only one CCD chip is used and the data from that chip are collapsed into a one-dimensional row to be read out at high speed. This allows a time resolution of $30 \mu \mathrm{s}$, and photon pile-up occurs only for count rates higher than $1500 \mathrm{~s}^{-1}$, much higher than the value observed during our observation (see Fig. 1). Using the ephemeris of Stewart et al. (1993) we find that the phase interval of the observation is 0.0110.017 , immediately after the phase-zero. We extract the energy spectrum in the energy band $0.7-10 \mathrm{keV}$. The continuum emission is fitted using a blackbody model absorbed by interstellar neutral matter and partially by neutral matter around the system. The 
TABLE 1. Continuum emission and absorption edges

\begin{tabular}{|c|c|c|c|}
\hline Parameters* & \multicolumn{3}{|c|}{ Parameters } \\
\hline $\mathrm{N}_{H}\left(\times 10^{22} \mathrm{~cm}^{-2}\right)$ & $1.22 \pm 0.08$ & $\mathrm{E}_{\mathrm{Si}}(\mathrm{keV})$ & 1.871 (fixed) \\
\hline $\mathrm{N}_{H_{p c}}\left(\times 10^{22} \mathrm{~cm}^{-2}\right)$ & $98_{-9}^{+5}$ & $\tau_{\mathrm{Si} \text { I }}$ & $0.46_{-0.03}^{+0.04}$ \\
\hline $\mathrm{f}_{p c}$ & $0.75 \pm 0.03$ & $\mathrm{E}_{\text {Si XIV }}(\mathrm{keV})$ & 2.673 (fixed) \\
\hline $\mathrm{kT}_{B B}(\mathrm{keV})$ & $1.21 \pm 0.02$ & $\tau_{\mathrm{Si} X I V}$ & $0.11_{-0.04}^{+0.03}$ \\
\hline $\mathrm{N}_{B B}$ & $0.025 \pm 0.001$ & $\mathrm{E}_{\mathrm{Ca} \text { I }}(\mathrm{keV})$ & 4.069 (fixed) \\
\hline $\mathrm{E}_{\mathrm{O} \text { VIII }}(\mathrm{keV})$ & 0.871 (fixed) & $\tau_{\mathrm{Ca} \mathrm{I}}$ & $<0.03$ \\
\hline$\tau_{\mathrm{O} \text { VIII }}$ & $0.72_{-0.38}^{+0.33}$ & $\mathrm{E}_{\mathrm{Fe} X X V}(\mathrm{keV})$ & 8.828 (fixed) \\
\hline $\mathrm{E}_{\mathrm{Ne} I \mathrm{X}}(\mathrm{keV})$ & 1.196 (fixed) & $\tau_{\mathrm{Fe} \mathrm{XXV}}$ & $<0.07$ \\
\hline$\tau_{\mathrm{Ne} \text { IX }}$ & $0.41_{-0.12}^{+0.14}$ & $\mathrm{E}_{\mathrm{Fe} \text { XXVI }}(\mathrm{keV})$ & $9.6_{-0.2}^{+0.5}$ \\
\hline $\mathrm{E}_{\mathrm{Ne} X}(\mathrm{keV})$ & 1.362 (fixed) & $\tau_{\mathrm{Fe} \text { XXVI }}$ & $0.09 \pm 0.08$ \\
\hline$\tau_{\mathrm{NeX}}$ & $0.12_{-0.07}^{+0.06}$ & & \\
\hline
\end{tabular}

* Uncertainties are at $90 \%$ confidence level for a single parameter; upper limits are at $95 \%$ confidence level. The threshold energy of the absorption edges are taken from Verner et al., 1996a

TABLE 2. Emission and absorption lines

\begin{tabular}{|c|c|c|c|c|c|}
\hline Ion* & $\begin{array}{c}\text { Measured E } \\
\text { keV }\end{array}$ & $\begin{array}{c}\text { Predicted } E^{\dagger} \\
\text { keV }\end{array}$ & Transition & $\begin{array}{c}\text { Width } \\
\text { eV }\end{array}$ & Flux ${ }^{* *}$ \\
\hline Ne $X$ & $1.01_{-0.04}^{+0.03}$ & 1.0218 & $1 s-2 p$ & 10 (fixed) & $2.8_{-1.8}^{+1.5}$ \\
\hline Mg XI & 1.3523 (fixed) & 1.3523 & $1 s^{2}-1 s 2 p$ & 10 (fixed) & $2.7_{-0.8}^{+0.0}$ \\
\hline Mg XII & 1.4723 (fixed) & 1.4723 & $1 s-2 p$ & 10 (fixed) & $2.6 \pm 0.6$ \\
\hline Si XIV & $1.979_{-0.004}^{+0.005}$ & 2.0054 & $1 s-2 p$ & $40 \pm 5$ & $9.7_{-1.0}^{+1.4}$ \\
\hline S XV & $2.42_{-0.02}^{+0.03}$ & 2.4607 & $1 s^{2}-1 s 2 p$ & $50_{-50}^{+40}$ & $2.5_{-0.7}^{+1.0}$ \\
\hline S XVI & $2.63 \pm 0.01$ & 2.6216 & $1 s-2 p$ & $40_{-30}^{+20}$ & $3.9_{-0.7}^{+0.8}$ \\
\hline Ar XVII & $3.11_{-0.01}^{+0.02}$ & 3.1398 & $1 s^{2}-1 s 2 p$ & $<20$ & $1.2 \pm 0.3$ \\
\hline Ar XVIII & $3.314 \pm 0.014$ & 3.3213 & $1 s-2 p$ & $<50$ & $1.3 \pm 0.3$ \\
\hline Ca XIX(r) & $3.9 \pm 0.2$ & 3.9023 & $1 s^{2}-1 s 2 p$ & $<60$ & $0.8_{-0.2}^{+0.3}$ \\
\hline Ca XX & $4.10_{-0.03}^{+0.01}$ & 4.1049 & $1 s-2 p$ & $<70$ & $0.8_{-0.2}^{+0.3}$ \\
\hline $\mathrm{Fe} \mathrm{I}$ & $6.39 \pm 0.01$ & 6.400 & - & $<50$ & $1.4 \pm 0.2$ \\
\hline Fe XXV(i) & $6.657 \pm 0.003$ & 6.6749 & $1 s^{2}-1 s 2 p$ & $40_{-8}^{+7}$ & $4.6 \pm 0.2$ \\
\hline Fe XXVI & $6.96 \pm 0.01$ & 6.9660 & $1 s-2 p$ & $<30$ & $1.1 \pm 0.1$ \\
\hline Ni XXVII(r) & $7.80 \pm 0.01$ & 7.7958 & $1 s^{2}-1 s 2 p$ & 10 (fixed) & $0.8 \pm 0.1$ \\
\hline Ni XXVIII & $8.16_{-0.07}^{+0.04}$ & 8.0871 & $1 s-2 p$ & 10 (fixed) & $0.3 \pm 0.1$ \\
\hline Fe XXVI & $\begin{array}{l}8.34_{-0.09}^{+0.08} \\
\end{array}$ & 8.2499 & $1 s-3 p$ & 10 (fixed) & $0.2 \pm 0.1$ \\
\hline Fe XXVI & $8.67 \pm 0.03$ & 8.6997 & $1 s-4 p$ & 10 (fixed) & $0.29 \pm 0.07$ \\
\hline Fe XXVI & $8.91 \pm 0.04$ & 8.9079 & $1 s-5 p$ & 10 (fixed) & $0.27 \pm 0.09$ \\
\hline Line $_{1} \neq$ & $5.6 \pm 0.1$ & - & - & $<80$ & $0.2_{-0.1}^{+0.8}$ \\
\hline Line $_{2} \S$ & $5.85_{-0.07}^{+0.30}$ & - & - & $<80$ & $-0.2 \pm 0.1$ \\
\hline
\end{tabular}

* Uncertainties are at $90 \%$ confidence level for a single parameter; upper limits are at $95 \%$ confidence level.

$\dagger$ The values are taken from Verner et al., 1996b

** $\left(\times 10^{-3}\right.$ photons $\left.\mathrm{cm}^{-2} \mathrm{~s}^{-1}\right)$

₹ Emission line not identified

$\S$ Absorption line not identified 


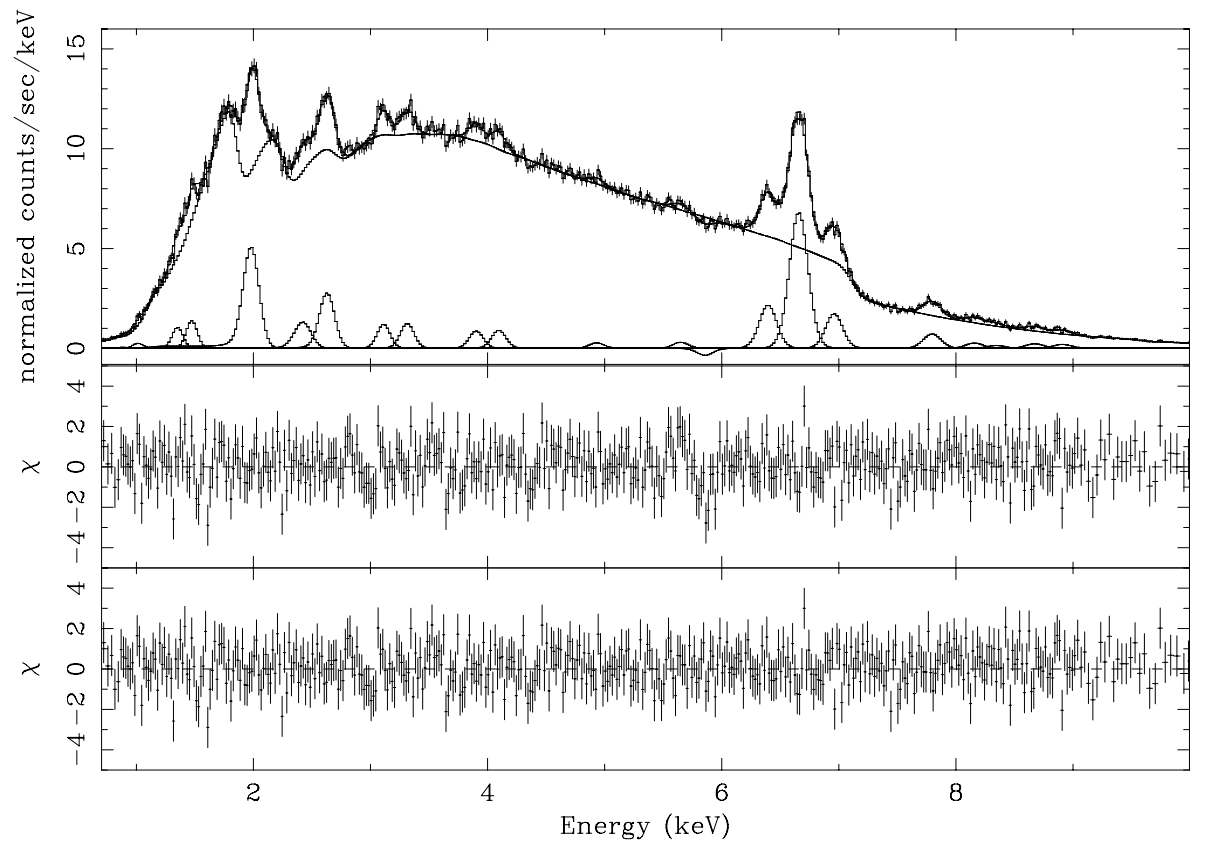

FIGURE 2. Top Panel: pn Spectrum of Cir $\mathrm{X}-1$ in the $0.7-10 \mathrm{keV}$ energy band (all the lines used to obtain the best fit are plotted). Middle Panel: Residuals after the addition of the identified absorption edges ans emission lines, a structure is evident between 5.5 and $6 \mathrm{keV}$. Bottom Panel: Residuals after the addition of an emission line at $5.6 \mathrm{keV}$ and an absorption line at $5.85 \mathrm{keV}$.

blackbody temperature and normalization are $1.2 \mathrm{keV}$ and 0.025 , respectively. The equivalent hydrogen column associated to the interstellar matter is $1.2 \times 10^{22} \mathrm{~cm}^{-2}$, the equivalent hydrogen column associated to the neutral matter embedding the system is around $1 \times 10^{24} \mathrm{~cm}^{-2}$, the covered fraction of the emitting surface is $75 \%$. We note a straordinary richness of absorption edges and emission lines in the residuals. Initially we add to the models the absorption edges keeping fixed the threshold energies (except that for Fe XXVI). We added the absorption edges associated to Si I, O VIII, Ne IX, Ne X, Si XIV, Ca I, Fe XXV, and Fe XXVI. The parameters of the continuum emission and the optical depths, $\tau$, associated to the absorption edges are reported in Tab. 1 . We find upper limits of the Ca I and Fe XXV optical depths, while a large value of $\tau$ associated to the $\mathrm{Si}$ I absorption edge indicating an overabundance of silicon.

As second step we add the emission lines to the model. Below $1.5 \mathrm{keV}$ we note the presence of three lines associated to $\mathrm{Ne}$ X, Mg XI, and Mg XII, since the low statistics between 0.7 and $1.5 \mathrm{keV}$ we keep fixed their centroids to the rest lab and the widths to 10 $\mathrm{eV}$ (the Mg XI centroid is fixed to the value of the resonance line). At the same way, since the low statistics above $7 \mathrm{keV}$, we keep fixed the widths of the emission lines identified between 7 and $10 \mathrm{keV}$. In this energy range we identify the Ni XXVII and Ni XXVIII emission lines, and the $\mathrm{Fe} X X V I \mathrm{Ly}_{\beta}, \mathrm{Ly}_{\gamma}$, and $\mathrm{Ly}_{\delta}$ emission lines. In the energy range between 2 and $7 \mathrm{keV}$ the line parameters are left free, we identify the Si XIV, S XV, 


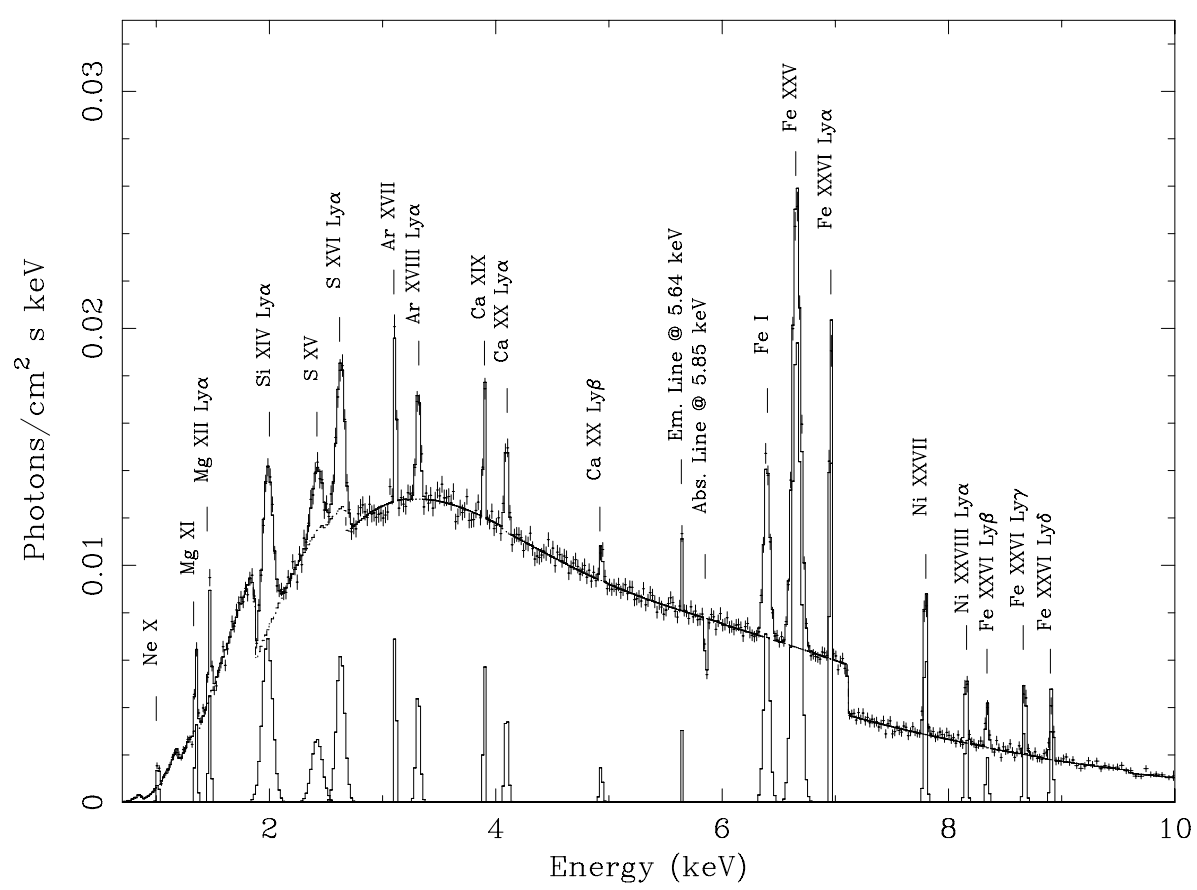

FIGURE 3. Unfolded spectrum of Cir X-1 corresponding to the best-fit model.

S XVI, Ar XVII, Ar XVIII, Ca XIX, Ca XX Ly $\alpha$, Ca XX Ly $\beta$, Fe I, Fe XXV, and Fe XXVI $\mathrm{Ly}_{\alpha}$. In Fig. 2 (middle panel) we plot the residuals corresponding to the model composed of the continuum emission, absorption edges, and emission lines above reported. In Tab. 2 we report the parameters of the lines. Looking at the residuals a structure is evident between 5.5 and $6 \mathrm{keV}$. We fit this localized structure adding an emission and an absorption line at 5.6 and $5.85 \mathrm{keV}$, respectively, although we do not identify them. The emission and the absorption line are detected at $2.9 \sigma$ and $3.9 \sigma$, respectively. In Fig. 2 (bottom panel) we plot the residuals after the adding of the lines between 5.5 and $6 \mathrm{keV}$, in Fig. 3 we plot the unfolded spectrum.

\section{PRELIMINARY DISCUSSION}

The $0.1-100 \mathrm{keV}$ extrapolated absorbed and unabsorbed flux is $5.2 \times 10^{-10}$ and $2.1 \times$ $10^{-9} \mathrm{erg} \mathrm{cm}^{-2} \mathrm{~s}^{-1}$, respectively. Adopting a distance to the source of $6.5 \mathrm{kpc}$ (Stewart et al., 1993) the extrapolated $0.1-100 \mathrm{keV}$ unabsorbed luminosity is $1 \times 10^{37} \mathrm{erg} \mathrm{s}^{-1}$. The corresponding blackbody radius is $6.2 \pm 0.3 \mathrm{~km}$.

The preliminary results of the fit indicate that near the zero phase $(0.011-0.017)$ neutral matter embeds the source confirming the results of previous works (e. g. Brandt et al., 1996). Our work seems to suggest the simultaneous presence of ionized and neutral matter probably located in two different region around the system. Brandt \& 
Shulz (2000) and Shulz \& Brandt (2002) detected the presence of P-Cygni profiles associated to highly ionized elements at similar phases, they assumed that the most plausible scenario producing these features was a wind moving along the radial direction above the accretion disc assuming the source almost edge-on. Looking at our results it is hard to sustain the edge-on scenario of $\mathrm{Cir} \mathrm{X}-1$ since in this case we cannot explain the presence of the prominent Fe I emission line, observed in the spectrum, requiring a lower inclination angle of the source. Our preliminary results seems to agree with the recent detection of a radio jet with angle separation between line of sight and jet direction of 5 degree (Fender et al., 2004) which implies a releasing of the edge-on scenario favoring a face-on one under the assumption that the radio-jet is directed almost perpendicularly to the equatorial plane. Finally we note another puzzling result, the continuum emission is well described using only a blackbody component. This result implies that we are observing only the emission from either the neutron star or the accretion disc because either one of the component is occulted or the accretion disc is not present. Both the scenarios are hard to be explained (assuming in the first case that the accretion disc is occulted) since it is natural to assume as mechanism producing the Fe I emission line the reflection from the disc. The remaining plausible scenario is the occulting of the neutron star but in this case it is anomalous the value of the inferred blackbody radius of $6 \mathrm{~km}$. Finally we note the marginal detection of a structure between 5.5 and $6 \mathrm{keV}$ modelled with an emission and an absorption line. The nature of this feature requires a more deep study that will be reported in a work in preparation.

\section{REFERENCES}

1. Begelman, M. C., McKee, C. F., Shields, G. A., 1983, ApJ, 271, 70

2. Brandt, W. N., \& Schulz, N. S., 2000, ApJ, 544, L123

3. Fender R., Wu K., Johnston H. et al., 2004, Nature, 427, 222

4. Iaria, R.; Burderi, L., Di Salvo, et al., 2001a, ApJ, 547, 412

5. Iaria, R., Di Salvo, T., Burderi, L., Robba, N. R., 2001b, ApJ, 561, 321

6. Jansen, F., Lumb, D., Altieri, B., et al. 2001, A\&A, 365, L1

7. Johnston, H. M., Fender, R., Wu, K., 1999, MNRAS, 308, 415

8. Murdin, P., Jauncey, D. L., Haynes, et al., 1980, A\&A, 87, 292

9. Shulz, N. S., \& Brandt, W. N., 2002, ApJ, 572, 971

10. Stewart, R. T., Caswell, J. L., Haynes, R. F., et al., 1993, MNRAS, 261, 593

11. Struder, L., Briel, U., Dennerl, K., et al. 2001, A\&A, 365, L18

12. Tauris, T. M.; Fender, R. P.; van den Heuvel, E. P. J.; et al., 1999, MNRAS, 310, 1165

13. Tennant, A. F., Fabian, A. C., Shafer, R. A., 1986a, MNRAS, 219,871

14. Tennant, A. F., Fabian, A. C., Shafer, R. A., 1986b, MNRAS, 221, 27P

15. Turner, M. J. L., Abbey, A., Arnaud, M., et al. 2001, A\&A, 365, L27

16. Verner, D. A., Ferland, G. J., Korista, K. T., et al., 1996a, ApS, 465, 487

17. Verner, D. A., Verner, E. M., Ferland, G. J., 1996b, Atomic Data and Nuclear Data Tables, 64, 1 
Copyright of AIP Conference Proceedings is the property of American Institute of Physics and its content may not be copied or emailed to multiple sites or posted to a listserv without the copyright holder's express written permission. However, users may print, download, or email articles for individual use. 